\title{
Acceptability of a Novel Vaginal MicrobicideDuring A Safety Trial Among Low-Risk Women
}

By Margaret E. Bentley, Kathleen M. Morrow, Andrew Fullem, Margaret A. Chesney, Scott D. Horton, Zeda Rosenberg and Kenneth H. Mayer

\begin{abstract}
Context: The increasing recognition that women who are unable or unwilling to discuss or use condoms with their sexual partners need female-controlled methods for preventing sexually transmitted diseases (STDs), including HIV, has led to considerable focus on the development of vaginal microbicides. While many such products are being tested for safety and effectiveness, clincal trials generally overlook another key factor in a product's impact on infection ratesits acceptability to users.
\end{abstract}

Methods: A Phase I clinical trial of a microbicidal gel included an assessment of the product's acceptability among 27 low-risk participants. Information on acceptability was gathered from structured interviews, participants' daily diaries and unstructured exit interviews.

Results: Participants reported only minor side effects of product use, such as itching, burning and difficulty urinating; two women developed candida infections while participating in the study. None of the side effects could be conclusively linked to use of the gel. Some women noted product discharge and messiness as drawbacks of the method, but this experience varied according to how often the women applied the gel. For example, one-third of those who used it once daily said that at least some of the time, it was too "wet or drippy," compared with two-thirds of women who inserted the gel twice a day. However, participants considered these "nuisance factors" that could be outweighed by the potential protective characteristics of the product. The majority reported that they would use the product if it were available and proven efficacious, and if they perceived that they were at risk of STD infection.

Conclusions: Additional testing of this product is urgently needed. Furthermore, as other products approach Phase I testing, acceptability assessments should be a key component of clinical trials.

Family Planning Perspectives, 2000, 32(4):184-188

$\mathrm{I}$ $n$ recent years, the testing and evaluation of topical microbicides to prevent sexually transmitted diseases (STDs), including HIV, have been the focus of considerable attention. This focus reflects increasing recognition that female-controlled methods are necessary to protect women who are unable or unwilling to discuss or use condoms with their sexual partners. ${ }^{1}$ A large, nationally representative survey of sexually active U.S. women reported that $80 \%$ would have been interested in using a vaginal microbicide product at some time during the past, and $40 \%$ would currently be interested, assuming that the product was protective against STDs. ${ }^{2}$ Although the results of that survey must be interpreted cautiously because of the limitations associated with asking hypothetical questions of "potential" users, they suggest a demand among U.S. women for female-controlled STD prevention methods.

There is clearly a need for such products in settings where, in a variety of ways, gender inequality places women at sexual risk. ${ }^{3}$ For example, in many geographic and sociocultural settings, women may be reluctant or unable to talk with their part- ners about the use of barrier methods for STD prevention. This is true in developed as well as developing countries, particularly among women who are disadvantaged by poverty or gender discrimination, who may not wish to compromise their relationships by questioning their partners' fidelity. ${ }^{4}$

Several dozen microbicide products are in various stages of development or testing. ${ }^{5}$ New products undergo lengthy, multiphased safety and toxicity studies before the Food and Drug Administration approves them for use in the United States. Preclinical studies evaluate a product's activity in either cell cultures or animal models. Once these studies have been completed, Phase I clinical studies begin; these trials are designed to assess safety in lowrisk populations. Phase II trials assess safety in higher-risk populations and test the biological plausibility of a given approach. Phase III studies evaluate the efficacy of the product in high-risk populations.

Additionally, products currently marketed for other purposes may be tested for efficacy in preventing STDs. A number of preparations containing nonoxynol-9 (an ingredient in many contraceptives) have been tested among high-risk populations in Phase II or III trials. While some of these products have been proven effective in preventing infection with several sexually transmitted pathogens, ${ }^{6}$ nonoxynol-9 film did not reduce the rate of HIV, gonorrhea or chlamydia infection in a large trial conducted in Cameroon. ${ }^{7}$

If a product is to have an impact on infection rates, it must be not only safe and effective in preventing STDs, but also acceptable to potential users. In this article, we

Margaret E. Bentley is associate chair and associate professor, Department of Nutrition, School of Public Health, and fellow, Carolina Population Center, University of North Carolina, Chapel Hill. Kathleen M. Morrow is assistant professor, Centers for Behavioral and Preventive Medicine, The Miriam Hospital and Brown University School of Medicine, Providence, RI. Andrew Fullem is director, HIV / AIDS Surveillance, Massachusetts Department of Public Health, Boston. Margaret A. Chesney is professor, Center for AIDS Prevention Studies, University of California at San Francisco. Scott D. Horton is data operations manager, Fred Hutchinson Cancer Research Center, Seattle. Zeda Rosenberg is scientific director, HIV Prevention Trials Network, Family Health International, Arlington, VA. Kenneth H. Mayer is professor, Brown University AIDS Program, Providence, RI, and Memorial Hospital, Pawtucket, RI. This work was supported by the HIV Network for Prevention Trials (HIVNET), sponsored by the National Institute of Allergy and Infectious Diseases, National Institutes of Health through contract N01-AI-35176 with Abt Associates; contract N01-AI-45200 with the Fred Hutchinson Cancer Research Center; and subcontracts with Memorial Hospital, Women and Infants Hospital, the Denver Public Health Department, Fenway Community Health Center, Howard Brown Health Center, New York Blood Center, New York University Medical Center, San Francisco Department of Public Health, University of Pennsylvania and University of Washington. ReProtect supplied BufferGel for the study. The authors are grateful for the dedication and commitment of participants, the efforts of site staff and the contributions of HIVNET community advisory board members. We thank the following individuals for their assistance: Fran Bettencourt, Susan Kelly and Amy Cooper conducted expert clinical and interview collection procedures; Rochelle Rosen and David Celentano conducted focus groups; Lynda Emel and Cliff Kelly provided data management and analysis assistance; Kevin Whaley and Thomas Moench provided insights for protocol development and interpretation; David Celentano and Kenrad Nelson collaborated on a uniform protocol for domestic and international Phase I studies Michael Gross and Ann Koonce contributed to the development of instruments; Ward Cates, Jr., Julie Welch, Leslie Hjeldness and staff at Family Health International assisted with protocol development and manuscript preparation; and Frances Dancy prepared the manuscript. This article does not necessarily reflect the views, policies or endorsement of the U.S. government. 
report on the acceptability to users of a novel vaginal microbicide, BufferGel, which is in the first stage of safety and toxicity testing.

The gel is designed to prevent disease transmission by promoting mild acidity ( $\mathrm{pH}$ less than five), because HIV and other STD pathogens are not viable outside the vagina's usual $\mathrm{pH}$ of around seven; in animal studies, the product has maintained the vaginal $\mathrm{pH}$ around 4.5 or lower. It also is intended to prevent conception and has sufficient buffer capacity to acidify approximately twice its own volume in human semen. The colorless, odorless gel is packaged in a six-inch white syringestyle applicator tube, which administers doses of $5 \mathrm{ml} .^{8}$

\section{Methods \\ Study Objectives and Design}

The study was conducted as part of the National Institutes of Health's HIV Prevention Trials Network, which evaluates the efficacy of different behaviorally linked biological HIV prevention strategies. One of the network's research priorities is the testing of candidate microbicides that have completed preclinical trials. This Phase I study was the first time that any women had mucosal exposure to this product, and thus the primary aim was to assess the gel's safety profilespecifically, to examine possible changes in vulvar and cervicovaginal mucosal health after seven and 14 days of daily or twice-daily exposure. Detailed safety and toxicity results are presented elsewhere. ${ }^{9}$ A secondary objective was to assess women's compliance with instructions for using the method and the product's acceptability to users.

The study was conducted at two hospitals in Rhode Island that are affiliated with Brown University; enrollment began in April 1997 and ended about six months later. Participants were recruited via multiple sources, including local public media (e.g., newspapers, cable television and public service announcements), university media, posted flyers and snowballing referrals from participants in ongoing HIV prevention studies. Women were eligible to participate if they were $18-45$ years old, had a regular menstrual cycle with a minimum of 18 days between menses and met the study criteria for being low-risk-that is, they had had no STD in the last six months; were sexually abstinent or were in a stable, monogamous relationship with a partner who was not known to be HIVpositive and was at low risk for HIV; and were not using injection drugs. To be eligible, women also had to be willing and able to give informed consent and to complete a daily study diary.

Participants agreed, for the duration of the study, to abstain from using injection drugs and vaginal products (such as lubricants, tampons, vaginal drying agents and douches), and to insert the microbicide as required by the protocol and immediately prior to intercourse. Sexually active participants agreed to have vaginal intercourse at least two times per week, using nonlubricated condoms provided by the study, while sexually abstinent participants agreed to continue to refrain from intercourse.

The study's protocol and ethical procedures were approved by university and hospital institutional review boards and the Office of Protection from Research Risks. Sexually active women were requested to discuss the study and its rules with their partner, but the male partners were not asked to provide informed consent, since they were not considered at risk (because of the requirement of condom use during sex).

\section{Procedures}

Enrolled women were instructed to insert $5 \mathrm{ml}$ of the gel into the vagina once daily for 14 days, beginning 3-5 days after menses. Following the completion of their next menses, they were given the option to "roll over" to a twice-daily regimen for an additional 14 days. Some participants did not wish to roll over and thus completed only the once-daily regimen; to ensure sufficient enrollment in the twicedaily regimen, the protocol permitted some participants to go directly to that phase. The protocol required participants to complete acceptability assessments at baseline and at days seven and 14, to maintain daily diaries, and to undergo four pelvic and two colposcopic examinations for each 14 days of enrollment. According to the protocol, eight in 10 sexually abstinent women had to complete the trial with no serious adverse events before the sexually active cohort began enrollment. Participants were paid $\$ 50$ for each study visit and $\$ 100$ every time they underwent colposcopy, for a minimum of $\$ 300$ and a maximum of $\$ 600$ for completion of the full study protocol.

Acceptability assessments were conducted through face-to-face structured interviews. The pretrial assessment explored previous vaginal product use and preconceived perceptions of acceptability. On the seventh day, all women were asked questions related to the product's characteristics (e.g., smell, consistency and color) and to the use of the applicator (e.g., ease of use, comfort, cleaning and disposability). On day 14, women who completed the trial were asked whether they would use this project if it was approved for vaginal use. For sexually active women, questions addressed the use of the product before, during and after sex, as well as their partner's reactions to it.

In the daily diary, participants were asked to record when and how often they inserted the product and whether they experienced any side effects, embarrassment or emotional reactions. Finally, unstructured exit interviews were completed with both women and their partners who chose to participate. Unstructured interviews allowed study participants to freely discuss their experience of completing a rigorous study, their experience with and perceptions about the microbicide, and reasons they would or would not use the product if it were available. Interviews were tape-recorded and transcribed for content analysis.

\section{Results \\ Study Participants}

In all, 27 women enrolled-16 who were sexually abstinent and 11 who were sexually active. Fifteen participants completed both the once-daily and the twicedaily application phases; the remaining 12 women completed only one phase. In the abstinent cohort, six women completed both phases, six completed oncedaily application and four completed twice-daily application. In the sexually active cohort, nine women completed both phases, one the once-daily application and one the twice-daily application.

Seventeen participants were white, three were black and seven were Hispanic. The women's ages ranged from 18 to 45 and averaged 31. Most participants had had at least a high school education, and more than half had completed at least some college.

Fourteen of the 27 women and two male partners chose to participate in the unstructured interviews. Three of these women were interviewed by telephone; the remainder participated in two focus groups. The male partners who participated provided informed consent.

\section{Safety and Side Effects}

Interview data suggested that no perceived side effects or symptoms caused major concern among the participants. This issue was directly probed during the interviews. Diary data, however, suggested that six sexually abstinent women and two sexually active participants experienced mild or moderate itching or burning with prod- 


\begin{tabular}{|c|c|c|c|c|c|c|}
\hline \multirow{2}{*}{$\begin{array}{l}\text { Characteristic and } \\
\text { frequency of } \\
\text { application }\end{array}$} & \multicolumn{2}{|l|}{ All } & \multicolumn{2}{|c|}{$\begin{array}{l}\text { Sexually } \\
\text { abstinent }\end{array}$} & \multicolumn{2}{|c|}{$\begin{array}{l}\text { Sexually } \\
\text { active }\end{array}$} \\
\hline & No. & $\%$ & No. & $\%$ & No. & $\%$ \\
\hline \multicolumn{7}{|l|}{$\begin{array}{l}\text { TOO WET/DRIPPY } \\
\text { Once daily }\end{array}$} \\
\hline Never/rarely & 15 & 68 & 8 & 67 & 7 & 70 \\
\hline Sometimes & 5 & 23 & 4 & 33 & 1 & 10 \\
\hline Often & 1 & 5 & 0 & 0 & 1 & 10 \\
\hline Always & 1 & 5 & 0 & 0 & 1 & 10 \\
\hline \multicolumn{7}{|l|}{ Twice daily } \\
\hline Never/rarely & 7 & 35 & 3 & 30 & 4 & 40 \\
\hline Sometimes & 6 & 30 & 5 & 50 & 1 & 10 \\
\hline Often & 4 & 20 & 1 & 10 & 3 & 30 \\
\hline Always & 3 & 15 & 1 & 10 & 2 & 20 \\
\hline \multicolumn{7}{|l|}{ TOO STICKY } \\
\hline Never/rarely & 17 & 77 & 9 & 75 & 8 & 80 \\
\hline Sometimes & 2 & 9 & 2 & 17 & 0 & 0 \\
\hline Often & 2 & 9 & 0 & 0 & 2 & 20 \\
\hline Always & 1 & 5 & 1 & 8 & 0 & 0 \\
\hline \multicolumn{7}{|l|}{ Twice daily } \\
\hline Never/rarely & 12 & 60 & 7 & 70 & 5 & 50 \\
\hline Sometimes & 4 & 20 & 1 & 10 & 3 & 30 \\
\hline Often & 2 & 10 & 1 & 10 & 1 & 10 \\
\hline Always & 2 & 10 & 1 & 10 & 1 & 10 \\
\hline \multicolumn{7}{|l|}{$\begin{array}{l}\text { SOILS CLOTHES } \\
\text { Once daily }\end{array}$} \\
\hline Never/rarely & 15 & 68 & 9 & 75 & 6 & 60 \\
\hline Sometimes & 6 & 27 & 2 & 17 & 4 & 40 \\
\hline Often & 1 & 5 & 1 & 8 & 0 & 0 \\
\hline Always & 0 & 0 & 0 & 0 & 0 & 0 \\
\hline \multicolumn{7}{|l|}{ Twice daily } \\
\hline Never/rarely & 9 & 45 & 4 & 40 & 5 & 50 \\
\hline Sometimes & 8 & 40 & 4 & 40 & 4 & 40 \\
\hline Often & 2 & 10 & 2 & 20 & 0 & 0 \\
\hline Always & 1 & 5 & 0 & 0 & 1 & 10 \\
\hline Total & na & 100 & na & 100 & na & 100 \\
\hline
\end{tabular}

Note: na=not applicable, because some women did not respond to all statements.
One-third of the 22 women who completed the once-daily regimen reported that the product was "wet/drippy" at least some of the time, compared with twothirds of the 20 women who inserted gel twice daily. (This may reflect the increased volume of product inserted in the twice-daily regimen.)

Most women noted that a vaginal discharge began after 1-2 days of product use. Many reported that the gel was clear when first administered but that the discharge was "thicker and lumpier" and white, resembling cottage cheese. Some were concerned that this discharge was a symptom of a yeast infection; others said that because they lacked other symptoms (e.g., itching, odor), they were not concerned.

Vaginal specimens taken on days seven and 14 of the study were examined microscopically for signs of yeast infection. In almost 80 woman-weeks of product exposure, one woman had a symptouct use, in most cases throughout the duration of the study. Three women (two abstinent and one sexually active) recorded difficulty urinating. One sexually active woman recorded a feeling of fullness with some pain during the last three days that she used the product twice daily.

Clinical data confirm the women's interview and diary reports. No women experienced serious side effects associated with use of the gel; two participants developed vaginal candida infections in the course of the study. ${ }^{10}$ In the absence of a control group, and given that none of the side effects were severe, the reported side effects could not be conclusively associated with product use.

\section{Acceptability: Product Characteristics}

Several women perceived the product to be "wet/drippy" or "sticky," although this experience varied depending on how often the women applied the gel (Table 1). indication that women were embarrassed by the discharge or that they had any emotional reactions to the product. One sexually active woman noted that she was "stressed," but this was not linked to her use of the gel.

\section{Acceptability: Effects on Sex}

In acceptability assessments, sexually active women were asked whether they agreed or disagreed with several statements about the gel's effects on their sexual pleasure. Two women who used the product once a day and one who used it twice daily reported that it interfered with intercourse. Five women from the oncedaily group and six who applied it twice daily - about half of each group-reported that it increased their sexual pleasure. About three-quarters in each group thought that their partner liked the way the gel felt.

Reflecting the lubricating effects of the product, one woman recorded in her diary that "sex was better during the study....it was wetter." However, three others recorded that although the gel was wet and lubricating at the beginning of sex, it soon became "dry" or "dry and sticky."

A number of issues related to the product's use during sex were covered in the focus-group discussions. Participants in these groups (including one of the two couples who participated) indicated that product discharge was present on the surface of the condom after sex. The wife of one couple, however, noted that after intercourse, the discharge was reduced. A woman whose partner was not present reported that while she had noticed the discharge, she could not recall her partner's commenting on it either during or after sex.

\section{Overall Evaluation}

At the 14-day assessment, women were asked, "If BufferGel were approved for vaginal use, would you use it?" Four of 14 abstinent women and four of 11 sexually active participants said they would not; two women did not complete the assessment. An open-ended question clarified the reasons that women would not use the product: They lacked a current sexual partner, had a monogamous partner, preferred a disposable applicator, found the product too messy, were able to negotiate condom use or did not perceive themselves to be at risk.

Focus-group participants were asked a similar question: "If this product was on the market, was available today, would you use it or recommend it to your daughter [or to] a friend?" Responses to this 
question were nearly all positive. Although some participants did not perceive themselves to be in need of such a microbicide because of their sexual behavior or perception of low risk, most thought that the product would be an important way for a woman to protect herself. One woman, however, thought there was an important role for a microbicide product yet said she would prefer other methods:

"There is something psychologically comforting about physical barriers... which doesn't happen with BufferGel....I don't have problems negotiating protection when I'm having sex."

Since product discharge was obviously an important issue, we probed whether the messiness or cottage cheese-like consistency would determine whether participants used the product if it were available. Some women responded that they would try such a product, but they would want to see what happened with the consistency and "clumpiness" during sex. Several women made the distinction between "real life" and their study experiences. They noted that in "real life," a woman would not be using the product every day or twice a day (although this generated interesting discussions about use among sex workers).

A male interviewer asked the two men who participated in the focus group, "If it came to the time that we thought this could go on to the next phase of the study, would you be willing to participate in a male safety study where you would expose the penis to the product directly?" One man responded that he would participate, since his partner had already been in the study; the other male did not respond to this question.

\section{Adherence}

The protocol called for the study to be replicated in four international sites if the product was found to be safe and acceptable; furthermore, the protocol could serve as a model for future Phase I microbicide trials. Consequently, information on participants' (and their partners') willingness and ability to complete the trial, with its intensive product use and follow-up requirements, was considered important. Interview, diary and focus-group data addressed these issues and allowed women (and men) to describe their experience in the trial.

One participant reported having broken a study rule. The woman had forgotten that she was not allowed to use tampons during the interval between once- and twice-daily use, but had discontinued tampon use as soon as she remembered. Although none of the sexually active couples were particularly happy about using condoms, they reported always using them. Several women noted that remembering to fill in the daily diaries was difficult. Adherence to the dosing schedule was virtually perfect, according to daily diary records, follow-up interviews and focus-group responses.

Although no woman had previously had a colposcopy, none reported that this was a problem. In fact, women were able to view the entire process on a video screen, and many reported that this was a valuable and interesting aspect of the examination. Several women expressed relief that they had had the opportunity to be "so thoroughly examined" and to know that they were "clean" and "healthy."

Women were queried about what had motivated them to enroll in the study. Several responded that they had desired to be part of "such a good cause" or to "give something back to society." In addition to responses reflecting altruism in general, some participants reported a specific desire to help women, since women are at increased risk for HIV infection. One participant reported that she always felt upset that women may contract the virus during one act of unprotected intercourse but that "it's not the same for the man." She commented:

"I was very excited knowing that [if women] choose to have sex with different men,...they can put something in them to protect themselves....So that's why through the whole thing I just thought about that-the power of women."

\section{Discussion}

To our knowledge, the study we have described was the first Phase I safety and toxicity vaginal microbicide trial to include in-depth assessments of product acceptability. It is not conventional to include a product acceptability component in a Phase I safety trial, but we believe that this approach is both sensible and feasible. By tracking use and acceptability early on, even among women who are at low risk relative to other populations, we can learn much about whether the product will be used in future clinical trials or if it becomes available in the marketplace.

The combination of qualitative and quantitative interviews allows assessment of variability across women regarding the product and its use during the trial. Phase I studies have limited numbers of participants, which makes statistical analysis and interpretation of quantitative data dif- ficult at best. The addition of qualitative data facilitates a fuller interpretation of the quantitative data.

There are some limitations of the study and its findings. First, because this study was designed primarily to assess the product's safety and toxicity, it required exaggerated doses; this could have a negative impact on acceptability. The limitations and potential bias of assessing acceptability among small groups of people during experimental testing have been discussed elsewhere. ${ }^{11}$

Second, we were unable to validate selfreports of product use. No specific measures were developed to address this concern, though we are confident that the gel was applied as required, partly because of the amount of it that clinical staff reported observing during pelvic and colposcopic examinations.

Third, because of the protocol design, women enrolled in this study had a low risk of acquiring HIV. They would not likely be a priority population for the marketing and use of vaginal microbicides. Product acceptability may differ among women who are at increased risk of disease acquisition. Also, condom use was required to prevent possible exposure to the product among participants' male partners. As such, no experiential information could be gathered about product acceptability during unprotected sex. Finally, we had difficulties recruiting male partners for focus groups and are unable to adequately discuss partners' reactions to the product.

So, what has been learned about this product and its potential acceptability as a vaginal microbicide? Most important, the low-risk women who participated in this safety trial reported that, given their perception of personal risk and the product's presumably reasonable affordability, they would use the gel to prevent STD infection. In view of this finding, and given that continued prevention efforts aimed at women are likely to heighten their perceived risk, this product has potential for significant acceptance among higher-risk women. The acceptability of microbicide products changes as the user's perspective changes. ${ }^{12}$ Therefore, higher-risk women may be even more motivated to try such a product. On the basis of their perceived risk, an estimated 20 million U.S. women would have some interest in using a microbicide product, depending on its specifications and cost. ${ }^{13}$ This market is likely to grow substantially as perceived risk converges with actual risk and as women learn more about the prevalence of viral STDs (especially her- 
pes and human papillomavirus).

Half of the women who used the product during sex reported that it enhanced sex, and nearly three-quarters reported that they thought their partners liked the feel of the gel during sex. This finding is similar to ones reported for other microbicide products, ${ }^{14}$ and it is perhaps one of the most promising results of all.

In its current formulation, this product has possible drawbacks. The most important of these is the messiness, stickiness or cottage cheese-like consistency of the product following application. Several women suggested that the product be reformulated into a suppository. It should be noted that the volume of gel required for this safety trial, especially during the twice-daily dosing regimen, could be greater than amounts that would be applied in actual use. Further acceptability studies of varying product volumes in higher-risk populations (e.g., including commercial sex workers or women in monogamous relationships with HIV-infected or high-risk partners) are needed to assess the required volume and the gel's associated physical attributes as potential barriers or facilitators of product use. Ideally, these studies would be done in the absence of condoms, to illuminate the relationship between product volume, physical attributes and semen.

As many women noted, if the product is proven efficacious, this could easily outweigh the "nuisance factors." Participants' comments throughout the study suggest that women will need to undergo extensive education about how they should optimally apply and use this product, and what they may experience with product use (e.g., discharge). Such education may enhance the product's acceptability, as women may be able to take measures in anticipation of product side effects (e.g., the use of panty liners for those concerned with leaking).

An important question that this study cannot answer, but that is perhaps key for all microbicides, is whether the gel could be used without the knowledge of a woman's partner. This may be important for women who risk abuse or the loss of a relationship when using a microbicide for protection. While we do not have adequate data to answer this question, our findings suggest that women are unlikely to be able to use this product clandestinely. A majority of the sexually active women and the two male partners interviewed observed that sex "felt different" and noted that the product may be discharged on the penis. However, a few women and both men noted that it would be more difficult to detect that sex was "different" if the partner was a casual partner. This is an important factor to examine in future microbicide research, especially as it pertains to women with multiple sexual partners.

To assess the relevance of these findings in international settings, Phase I studies of the product, including similar assessments of its acceptability, have been undertaken in India, Malawi, Thailand and Zimbabwe. Key research questions at international sites include the product's affordability and accessibility, how preintroduction educational strategies may facilitate product acceptance and, of course, whether women will be able to use the product in privacy and without partner knowledge, if necessary. (Stability testing is not part of the international trials.) Cultural beliefs and current sexual practices of women and men who are at risk will be examined.

The positive safety and toxicity results of the trial, ${ }^{15}$ along with the product's acceptability, point up an urgent need to move ahead with additional testing. As other vaginal microbicide products become ready for Phase I testing, it is important that acceptability assessments be considered a key part of clinical trials.

\section{References}

1. Gupta GR and Weiss E, Women and AIDS: Developing a New Health Strategy, International Center for Research on Women (ICRW) Policy Series, Washington, DC: ICRW, 1993; Heise L and Elias C, Transforming AIDS prevention to meet women's needs: a focus on developing countries, Social Science and Medicine, 1995, 40(7):931-943; Stone $\mathrm{AB}$ and Hitchcock PJ, Vaginal microbicides for preventing the sexual transmission of HIV, AIDS, 1994,
8(1):285-293; and Elias CJ and Coggins C, Female-controlled methods to prevent sexual transmission of HIV, AIDS, 1996, 10(3):543-551.

2. Darroch JE and Frost JJ, Women's interest in vaginal microbicides, Family Planning Perspectives, 1999, 31(1):16-23.

3. Stein ZA, HIV prevention: the need for methods women can use, American Journal of Public Health, 1990, 80(4):460-462; and Elias CJ and Heise LL, Challenges for the development of female controlled vaginal microbicides, AIDS, 1994, 8(1):1-9.

4. Sobo EJ, Choosing Unsafe Sex: AIDS-Risk Denial Among Disadvantaged Women, Philadelphia: University of Pennsylvania Press, 1995

5. Ibid.; Alliance for Microbicide Development, Microbicides: research and development status as of September 1998, Takoma Park, MD: Alliance for Microbicide Development, 1998; and Rosenthal SL et al., Topical microbicides: current status and research considerations for adolescent girls, Sexually Transmitted Diseases, 1998, 25(7):368-377.

6. Kreiss J et al., Efficacy of nonoxynol-9 contraceptive sponge use in preventing heterosexual acquisition of HIV in Nairobi prostitutes, Journal of the American Medical Association, 1992, 268(4):477-482; and Martin H et al., Safety of nonoxynol-9 vaginal gel in Kenyan prostitutes, Sexually Transmitted Diseases, 1997, 24(5):279-283.

7. Roddy RE et al., A controlled trial of nonoxynol 9 film to reduce male-to-female transmission of sexually transmitted diseases, New England Journal of Medicine, 1998, 339(8):504-510.

8. ReProtect, BufferGel Investigator's Brochure 1997, Baltimore: ReProtect.

9. Mayer KH, Peipert J and Fleming T, The safety and tolerability of BufferGel, a novel vaginal microbicide studied in U.S. women, Clinical Infectious Diseases, 2000 (forthcoming).

10. Ibid.

11. Keller A, Contraceptive acceptability research: utility and limitations, Studies in Family Planning, 1979, 10(8/9):230-237.

12. Heise L, Beyond Acceptability: Reorienting Research on Contraceptive Choice, London: Reproductive Health Matters, 1997.

13. Darroch JE and Frost JJ, 1999, op. cit. (see reference 2).

14. Steiner M, Spruyt A and Joanis C, Acceptability of spermicidal film and foaming tablets among women in three countries, International Family Planning Perspectives, 1995, 21(3):104-107.

15. Mayer KH, Peipert J and Fleming T, 2000, op. cit. (see reference 9). 